\title{
Gender Identification in Human Gait Using Neural Network
}

\author{
Richa Shukla \\ Dept. of Computer Science, SRCEM, Banmore, Morena, India \\ Email: richa_9_s@yahoo.co.in \\ Reenu Shukla \\ Dept. of Computer Science, Oriental University, Indore, India
}

Anupam Shukla

Dept. of Computer Science, ABV- Indian Institute of Information Technology and management, Gwalior, India

Sanjeev Sharma

Dept. of Computer Science, ABV- Indian Institute of Information Technology and management, Gwalior, India

Nirupama Tiwari

Dept. of Computer Science, SRCEM, Banmore, Morena, India

\begin{abstract}
Biometrics is an advanced way of person recognition as it establishes more direct and explicit link with humans than passwords, since biometrics use measurable physiological and behavioural features of a person. In this paper gender recognition from human gait in image sequence have been successfully investigated. Silhouette of 15 males and 15 females from the database collected from CASIR site have been extracted. The computer vision based gender classification is then carried out on the basis of standard deviation, centre of mass and height from head to toe using Feed Forward Back Propagation Network with TRAINLM as training functions, LEARNGD as adaptation learning function and MSEREG as performance function. Experimental results demonstrate that the present gender recognition system achieve recognition performance of $93.4 \%, 94.6 \%$, and $94.7 \%$ with 2 layers/20 neurons, 3 layers/30 neurons and 4 layers/30 neurons respectively. When the performance function is replaced with SSE the recognition performance is increased by $2 \%, 2.4 \%$ and $3 \%$ respectively for 2 layers/20 neurons, 3 layers/30 neurons and 4 layers $/ 30$ neurons. The above study indicates that Gait based gender recognition is one of the best reliable biometric technology that can be used to monitor people without their cooperation. Controlled environments such as banks, military installations and even airports need to quickly detect threats and provide differing levels of access to different user groups.
\end{abstract}

Index term - Gender Recognition, Gait, Silhouette, Feature extraction, Neural network

\section{INTRODUCTION}

Gait recognition is the process of identifying an individual by a particular way or manner in which they walk. Without any interaction or co-operation from the subject, gait offers the possibility to identify people at a distance in less unobtrusive biometric way. This is the most important property which makes it so attractive. Human gait recognition as a new biometric aimed to recognize person via the style of people walking, which contain the physiological or behavioural characteristics of human. Gait-based gender classification is still immature because of its unique advantages of being noncontact, non-invasive, and easily acquired at a distance, it is gaining increasing interest from researchers.

Human Gait classification and recognition giving some advantage compared to other recognition system. Gait classification system does not require observed subject's attention and assistance. It can also capture gait at a far distance without requiring physical information from subjects [1-3]. There is a significant difference between human gait and other biometrics classification. In human gait, we should use video data instead of using image data as other biometrics system used widely. In video data, we can utilize spatial data as well as temporal data compare to image data. Most of the gait classification and or recognition system created are using spatial data only [4-11].Human Gait Gender Classification as a recognition system divided in three main subject; preprocessing, feature extraction and classification. In most gait recognition approaches [1214] recognition features are extracted from silhouette images. Dimosthenis Ioannidis et.al. (2007) [15] proposed an innovative gait identification and authentication method based on the use of novel 2-D and 3-D features. The human silhouette is segmented into seven components, namely head, arm, trunk, thigh, front-leg, back-leg, and feet. The leg silhouettes for the front-leg and the back-leg are considered separately because, during walking, the left leg and the right leg are in front or at the back by turns. Each of the seven components and a number of combinations of the 
components are then studied with regard to two useful applications: human identification (ID) recognition and gender recognition. Many social interactions depend greatly on correct gender perception. If a computer based biometric system can recognize gender, it will be very helpful in many applications. For example, gender classification can improve surveillance systems, intelligence, analyse customers for store managers, allow robots to perceive gender, etc. Automatic gender classification can be based on face [16-19] or gait [20]. A novel multimodal biometric recognition system using three modalities including face, ear and gait, based on Gabor+ PCA feature extraction method with fusion at matching score level is done by Ali Pour Yazdanpanahet. al. (2010) [21].

This paper presents gender identification using gait biometrics method based upon silhouette analysis measured during walking. Recognizing people by gender depends greatly on how the silhouette shape of an individual changes over time in an image sequence. So, we may consider gait motion to be composed of a sequence of static body gesture and expect that some distinguishable signatures with respect to those static body poses can be extracted and used for recognition.

The rest of the paper is organized as follows. Section II describes the proposed method in which silhouette extraction, feature extraction process is explained. Section III explains the overall implementation and experiment of gait recognition system. Finally, we conclude in section IV.

\section{PROPOSED METHOD}

In this study, we use CASIA Gait Dataset of Class B using one perpendicular camera. The Class B Dataset consists of 124 different people. Feature is extracted by creating silhouettes, fortunately we did not have to create silhouettes because CASIA provide silhouettes image in their dataset. We have taken 30 silhouettes of female and male from CASIA Gait Database (Dataset B).

\section{A. Silhouette extraction}

All subjects walking naturally in a straight line along with the side view at 90 degree are chosen in our work. In biometric gait recognition system the database is stored in video form and then sequences of frame of a walking person is created. These frames are converted into silhouette. Silhouette extraction and features extraction are important pre-processing tasks in gait recognition system. Silhouette from the frame can be extracted using the image processing operation.

Silhouette extraction algorithms rely on a known background. The motional individual silhouette must be detected before getting the gait feature. The background image is evaluated by removing the object from the picture. To find silhouette from the image background subtraction is a new approach and relatively simple. Background from the image sequence of a walking people is obtained; Mean image is computed by averaging the gray-level at each pixel over the entire

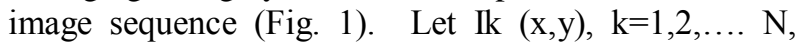
represent sequence of $\mathrm{N}$ images. After that background subtraction is applied to extract moving object. To improve the quality of extracted silhouette, image processing operation is applied.

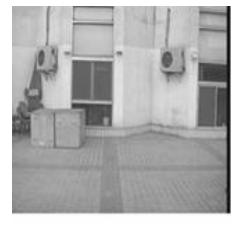

a

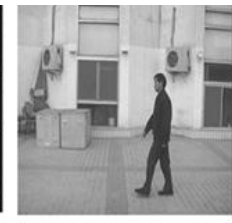

b

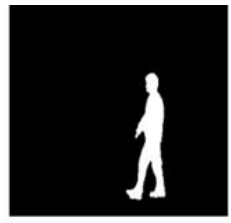

$\mathrm{c}$
Figure 1.Gait detection. (a) Background image; (b) Original image and (c) Extracted silhouette

\section{B. Feature Extraction}

Since now we have database in silhouette form, silhouette show most of the body part so we can extract feature from this silhouette. Two basic feature extraction techniques are classified as feature based and holistic method [22]. A feature vector is one method to represent feature of image, or part of an image (an object), by carrying out measurements on a set of features. The feature vector is an $\mathrm{n}$ dimensional vector that contains these measurements. This vector can be used to classify an object or to provide condensed higher-level image information. A feature is robust if it provides consistent results across the entire application domain. Holistic feature extraction technique find feature with reduced dimensionality by projecting original data onto the basic vectors and these extracted features can improve classification performance by reducing irrelevant feature from the data set.

The images we analyze are stored in the form of a two-dimensional data array, in which each datum is referred to as a pixel (picture element). We refer an individual pixel located at row $\mathrm{i}$ and column $\mathrm{j}$ by the notation [23]. $B(i, j)=$ the brightness of the image at the point $(i, j)$.

At the time of walking, the center of mass of human body changes from instance to instance so we are using center of mass as a feature. Center of mass shows brighten weighted average of $\mathrm{x}$ and $\mathrm{y}$ coordinates pixels in the frame. Center of mass of the white pixels area for binary images is the same as center of mass, if we consider the intensity at a point as the mass of that point. We can calculate centre of mass coordinate in binary image by using following formula.

$$
\begin{aligned}
& \bar{x}=\frac{\sum_{i=0}^{n} \sum_{j=0}^{m} j \cdot B(i, j)}{A} \\
& \bar{y}=\frac{\sum_{i=0}^{n} \sum_{j=0}^{m} i \cdot B(i, j)}{A}
\end{aligned}
$$


Here $\mathbf{x}$ and $\mathbf{y}$ are center of mass points in image. Here $\mathrm{m}$ and $\mathrm{n}$ is dimension of matrix which store image in matrix form. A is defined as area of region and can be calculated by following formula:

$$
A=\sum_{i=0}^{n} \sum_{j=0}^{m} B(i, j)
$$

Second feature is Standard deviation. It can be defined as an average grey value within the selection and sum of the grey values of all the pixels in the selection divided by the number of pixels. For RGB images, the mean has been calculated by converting each pixel to gray scale using the formula:

gray $=0.299$ red +0.587 green +0.114 blue

or

the gray $=($ red + green + blue $) / 3$

Standard deviations of the grey values are used to generate the mean grey value.

Third Feature is Height from head to toe as shown in Fig. 2. As height is one of the feature that differentiate between male and female, generally Male has more height than female.

\section{IMPLEMENTATION AND EXPERIMENTS}

The proposed Algorithm for Gender recognition using gait is shown in Fig. 3. Firstly the database is collected using camera.

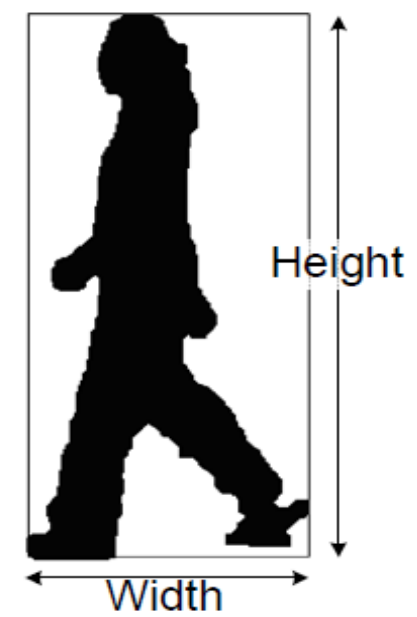

Figure 2.Boundary box to measure width

After that, silhouette is created for segmented image by motion detection and segmentation methods that are applied to detect and segment a walking person in a given video.

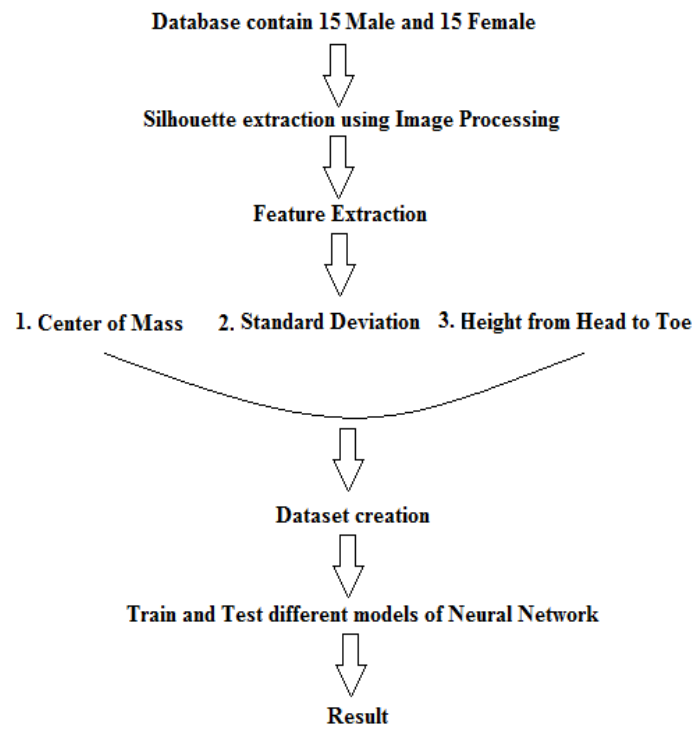

Figure 3. Representation of testing and training data

Image processing methods are applied to extract the feature for each silhouette and these features represent the special characteristics of walking person. It is shown by the feature vector. Finally Dataset is created of extracted feature used for identification.We will implement the proposed methods to the CASIA (Chinese Academy of Sciences) Gait Database. CASIA Gait dataset has four class datasets: Dataset A, Dataset B (multi-view dataset), Dataset C (infrared dataset), and Dataset D (foot pressure measurement dataset). We have used B class dataset in 90 degrees point of view.

As classifier, neural network is used with feed forward back propagation. In this paper we used TRAINLM (Levenberg-Marquardt algorithm) as training function, LEARNGD (Gradient descent weight/bias) as Adoption Function and MSEREG (Mean squared error and the mean squared weight and bias) as Performance function. This combination is denoted as Data set I. Better accuracy of gender recognition is achieved when MSEREG (Mean squared error and the mean squared weight and bias) is replaced by SSE (Sum squared error) as Performance Function while keeping others same as mentioned above. It is denoted as Data set II. The Data set II i.e. (MSEREG replaced by SSE as Performance Function) shows better recognition rate of $97.7 \%$ with 4 layers and 30 neurons in comparison of Data set I.

\section{A. Training and Testing Datasets}

This paper uses the ANN architecture feed forward back propagation learning algorithm to create, train and test the neural network for gender recognition using gait with MATLAB software as neural network toolbox. The original data sets contains 1600 instance of 30 subjects including male and female where each subject has 50 sequence instances. This data set groups into different data sets as shown in table I. Each set is divided into two subsets i.e training set and testing set. 
TABLE I DIFFERENT DATA SETS USED IN THE PROPOSED STUDY

\begin{tabular}{|c|c|c|c|c|}
\hline $\begin{array}{c}\text { Data set Group } \\
\text { name }\end{array}$ & $\begin{array}{c}\text { Training set } \\
\text { percentage }\end{array}$ & $\begin{array}{c}\text { Testing set } \\
\text { percentage }\end{array}$ & Training instance & Testing Instance \\
\hline Data set1(DS1) & $90 \%$ & $10 \%$ & $\begin{array}{c}\text { Male }-720 \\
\text { Female-720 }\end{array}$ & $\begin{array}{c}\text { Male-80 } \\
\text { Female-80 }\end{array}$ \\
\hline Data set 2(DS2) & $80 \%$ & $20 \%$ & $\begin{array}{c}\text { Male-640 } \\
\text { Female-640 }\end{array}$ & $\begin{array}{c}\text { Male-160 } \\
\text { Female-160 }\end{array}$ \\
\hline Data set 3(DS3) & $70 \%$ & $30 \%$ & $\begin{array}{c}\text { Male-560 } \\
\text { Female-560 }\end{array}$ & $\begin{array}{c}\text { Male-240 } \\
\text { Female-240 }\end{array}$ \\
\hline
\end{tabular}

\section{B. Computational Analysis}

In the study of 15 subjects of female and male each is collected from CASIA Gait Database [24]. Dataset B is used, where all subjects are walking naturally in a straight line along with the side view at 90 degree are chosen in our work that is from right to left. Each subject has 50 frames for training and testing. Matlab has been used to carry out the experiment. Feed forward back propagation neural network are used for training and testing. The Data set is divided in three groups DS1, DS2, and DS3 for Dataset I and Dataset II as shown in Table 1. The result presented in Fig 4(a) indicate that features used above along with the changes in the function of Performance, training and learning shows effective results to identify people from distance. Training function (Fig4(b)) are mathematical procedures used to automatically adjust the network's weights and biases. Performance graph (Fig 4c)) shows network's performance according to the mean of squared errors by training, validation and testing of data.Data is train and tested on feed forward back propagation networks with change in algorithm for training, learning and performance function. Here we used TRAINLM
Levenberg - Marquardt algorithm as applied to neural networks to adjust the weights as training function. TRAINLM is one of the fastest training algorithms available with training being 10 - 100 times faster than simple gradient descent back - propagation of error and it also provides an estimate of how many weights and biases are being effectively used by the network.

LEARNGD is used as a learning function. LEARNGD is the gradient descent weight/bias learning function and calculates the weight change $\mathrm{dW}$ for a given neuron from the neuron's input $\mathrm{P}$ and error $\mathrm{E}$, and the weight (or bias) learning rate LR.

According to the gradient descent: $\mathrm{dw}=1 \mathrm{r}^{*} \mathrm{gW}$.

Lastly MSEREG is used as a network performance function. It measure performance as a weight sum of two factors: one is mean squared weight and bias values having recognition rate of $94.7 \%$ (fig.4). For more effective and accurate recognition, performance function has been changed from MSEREG to SSE and it shows better recognition rate of $97.7 \%$ (Fig.5). SSE is a network performance function and measure performance by sum of squared error.

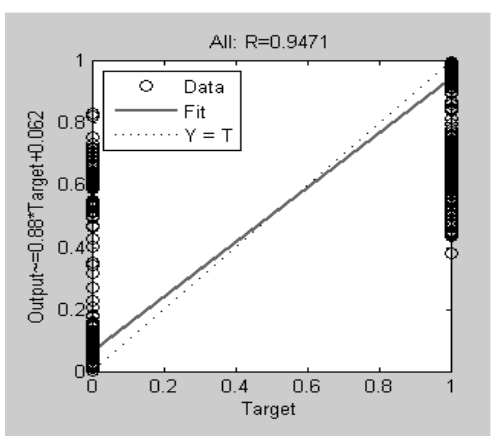

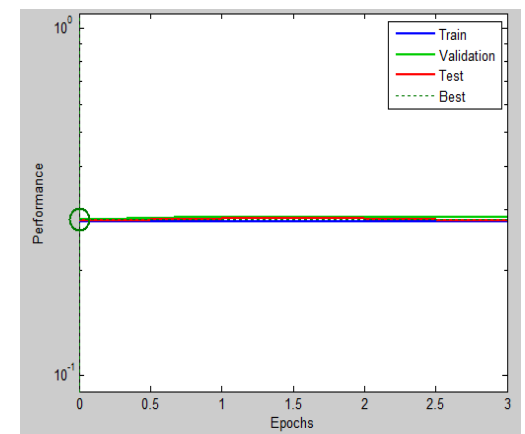

b

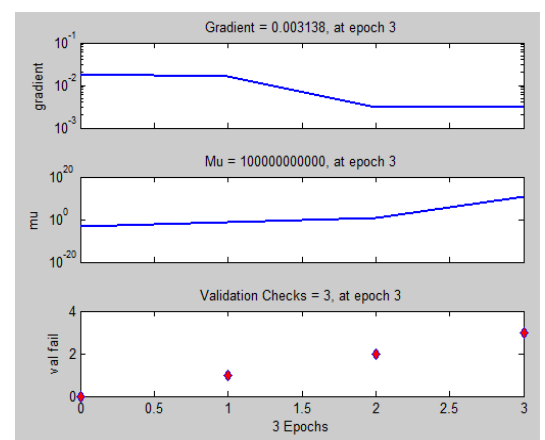

c

Figure 4. Data set I having 4 layers and 30 neurons (a) Regression graph, (b)Performance graph and (c)Training State 


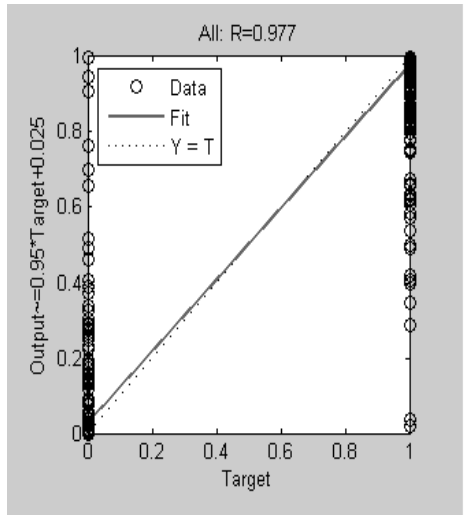

a

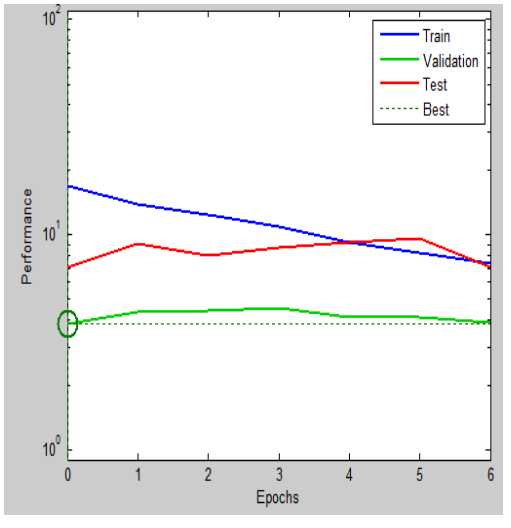

b

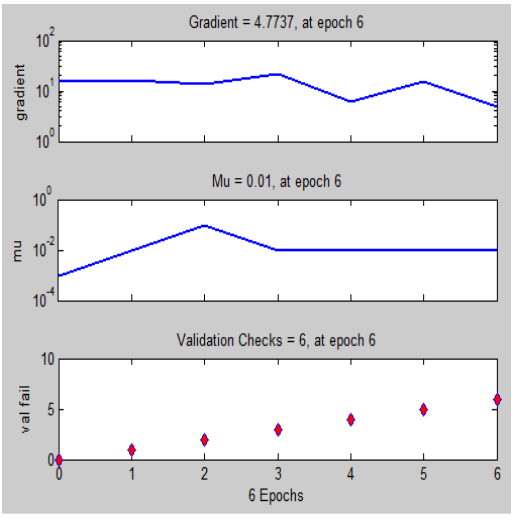

c

Figure 5. Data set II having 4 layers and 30 neurons (a) Regression Graph, (b) Performance graph and (c)Training state

TABLE II COMPARATIVE ANALYSIS

\begin{tabular}{|c|c|c|c|c|}
\hline Data set name & Network & $\begin{array}{l}\text { Number of hidden } \\
\text { layers and neurons }\end{array}$ & $\begin{array}{c}\text { Classification } \\
\text { accuracy }\end{array}$ & Epoch \\
\hline \multirow{3}{*}{$\begin{array}{l}\text { Data set I } \\
\text { TRAINLM, } \\
\text { LEARNGD \& } \\
\text { MSEREG }\end{array}$} & \multirow{3}{*}{$\begin{array}{l}\text { Feed forward } \\
\text { back } \\
\text { propagation }\end{array}$} & 2 and 10 & 93.4 & 13 \\
\hline & & 3 and 20 & 94.6 & 11 \\
\hline & & 4 and 30 & 94.7 & 3 \\
\hline \multirow{3}{*}{$\begin{array}{l}\text { Data set II } \\
\text { TRAINLM, } \\
\text { LEARNGD \& } \\
\text { SSE }\end{array}$} & \multirow{3}{*}{$\begin{array}{l}\text { Feed forward } \\
\text { back } \\
\text { propagation }\end{array}$} & 2 and 10 & 95.4 & 14 \\
\hline & & 3 and 20 & 97.0 & 11 \\
\hline & & 4 and 30 & 97.7 & 6 \\
\hline
\end{tabular}

\section{IV.CONCLUSION AND FUTURE WORK}

This paper presents gender recognition using gait by specific features, like center of mass, standard deviation and height from head to toe. Feed forward back propagation network is being used to identify gender by using TRAINLM, LEARNGD \& MSEREG as training, learning and performance function and result shows recognition rate of $94.71 \%$ with 4 layers and 30 neurons. When performance function MSEREG has been replaced by SSE, better recognition rate of $97.7 \%$ is achieved. The work can be further extended to develop new multimodal biometric [25] system where gait can be combined with other biometrics system.

\section{REFERENCES}

[1] N. V. Boulgouris, D. Hatzinakos, and K. N. Plataniotis, "Gait recognition: a challenging signal processing technology for biometric identification,'IEEE Signal Processing Magazine, vol. 22, pp. 78-90, 2005.

[2] M. S. Nixon and J. N. Carter, "Automatic Recognition by Gait,"Proceedings of the IEEE, vol. 94, pp. 2013-2024, 2006.

[3] Y. Jang-Hee, H. Doosung, M. Ki-Young, and M. S. Nixon, "Automated Human Recognition by Gait using Neural Network,"First Workshops on Image Processing Theory, Tools and Applications, 2008, pp. 1-6.

[4] J. Wang, M. She, S.Nahavandi, and A.Kouzani, “A
Review of Vision-based Gait Recognition Methods for Human Identification," IEEE Computer Society, 2010 International Con-ference on Digital Image Computing: Techniques and Applications, pp. 320 327,2010

[5] B. Pogorelc and M. Gams, "Medically Driven Data Mining Application: Recognition of Health Problems from Gait Patterns of Elderly,"IEEE International Conference on Data Mining Workshops, 2010.

[6] S. Ha, Y. Han and H. Hahn, "Adaptive Gait Pattern Generation of Biped Robot based on Human's Gait Pattern Analysis,"World Academy of Science, Engineering and Technology, 34, 2007.

[7] M. Hu, Y. Wang, Z. Zhang and Y. Wang, "Combining Spatial and Temporal Information for Gait Based Gender Classification," International Conference on Pattern Recognition 2010.

[8] X. Li, J. Stephen, M. S. Yan, D. Tao and D.Xu, "Gait Components and Their Application to Gender Recognition, IEEE Transactions On Systems, Man, And Cybernetics-Part C:"Applications And Reviews, 38(2),2008.

[9] S. Yu, T. Tan, Kaiqi Huang, KuiJiaand Xinyu Wu, "A Study on Gait-Based Gender Classification," IEEE Transactions On Image Processing, 18(8), 2009.

[10] M.Hanmandlu, R.Bhupesh Gupta, F. Sayeed and A.Q. Ansari“An Experimental Study of different 
Features for Face Recognition,'International Conference on Communication Systems and Network Technologies, 2011.

[11] R. Asmara, A.Basuki and K. Arai, "A Review of Chinese Academy of Sciences (CASIA) Gait Database As a Human Gait Recognition Dataset," Industrial Electronics Seminar, 2011, Surabaya Indonesia.

[12] J. P. Foster, M. S. Nixon andA. Prudel-Bennett, "Automatic gait recognition using area-based metrics,"Pattern Recognition Letters, 24, 24892497, 2003.

[13] P. J. Phillips, S. Sarkar, I. Robledo, P. Grother and K. Bowyer, 2002. "The gait identification challenge problem: Data sets and baseline algorithm,"In:Proc. of 16th Internat. Conf. on Pattern Recognition, ICPR, 1, 385-388, 2002.

[14] K. Kale, N. Cuntoor, B. Yegnanarayana, A. N. Rajagopalan and R. Chellappa, "Gait analysis for human identification," In:Proc. of the 3rd Internat. Conf. on Audio and Video Based Person Authentication, 2003.

[15] D. Ioannidis, D. Tzovaras, S. DamousisArgyropoulos, K. Moustakas, "Gait Recognition Using Compact Feature Extraction Transforms and Depth Information,"IEEE Trans Inf Forensics Security, 2(3), 623-630, 2007.

[16] Golomb, B.A., Lawrence, D.T., Sejnowski, T.J., 1991. SexNet: A neural network identifies sex from human faces. NIPS 3 In:Proc. of Internat. Conf. Advances in Neural Information Processing Systems, NIPS 3, Vol . 3, pp.572-577.

[17] B. Moghaddam, M. H. Yang, 2002. "Learning gender with support faces,"IEEE Trans Pattern Anal Mach Intell., (PAMI), 24(5), 707-711, 2002.

[18] Y. Andreu, P. Garc'ia-Sevilla, A. Ram'on, E. Mollineda, 2009. "Dealing with Inaccurate Face Detection for Automatic Gender Recognition with Partially Occluded Faces,"In:Proc. of the 14th Iberoamerican Conf. on Pattern Recognition: Progress in Pattern Recognition, Image Analysis, Computer Vision, and Applications,BayroCorrochano and J.-O. Eklundh (Eds.), CIARP 2009, LNCS 5856, Springer-Verlag Berlin Heidelberg.5856, 749-757, 2009.

[19] H. Harb, L. Chen, 2003. "Gender identification using a general audio classifier,"In:Proc. of Internat. Conf. on Multimedia and Expo, ICME'03," 1, 733-736, 2003. Washington, DC, USA.

[20] X. Li, S. J. Maybank, S. Yan, D. Tao and D. Xu, "Gait components and their application to gender recognition,"IEEE Trans. Syst., Man, Cybern. C, Appl. Rev., 38(2), 145-155, 2008.

[21] A. P.Yazdanpanah, K. Faez, R. Amirfattahi, 2010. "Multimodal Biometric System Using Face, Ear
And Gait Biometrics,"In: Proc. of 10th Internat. Conf. on Information Science, Signal Processing and their Applications, ISSPA, 251-254, 2010.

[22] J. H. Na, M. S. Park and J. Y. Choi, "Pre-clustered principal component analysis for fast training of new face databases," In: Proc. of Internat. Conf. on Control, Automton. Syst., ICCAS”07, 1144-1149, 2007.

[23] S. E. Umbaugh, Y. Wei and M. Zuke,"Feature extraction in image analysis". IEEE Eng Med Bio Soc., 16(4), 62-73, 1997

[24] [http://www.cbsr.ia.ac.cn/english/Gait\%20Database s.asp].

[25] A. Ross, A. Jain, "Information fusion in biometrics,"Pattern Recognition Letters, 24(13), 2115-2125, 2003.

Richa Shukla is a student of final year of M.Tech in Computer Science from SRCEM , Banmore. She received her B.E. degree in Computer Science from Institute of Technology and Management, Gwalior. Her area of research is Soft Computing, Biometrics and Image Processing.

Reenu Shukla is a student of final year of M.Tech in Computer Science from Oriental University, Indore. She received her M.C.A degree from Institute of Technology and Management, Gwalior. Her area of research is Soft computing, Biometrics and Image Processing.

Prof. Anupam Shukla is the Professor in the ICT Department of ABVIndian Institute of Information Technology and Management Gwalior. $\mathrm{He}$ is the Inchrge of Soft Computing and Expert System Laboratory at the Institute. He received his Ph.D. in Electronics \& Telecommunication Engineering in 2002 from NIT Raipur. He has 22 years of teaching experience. He has published around 90 papers in various national and international journals/conferences. $\mathrm{He}$ is the Editor and Reviewer in various journals. He received Young Scientist Award from Madhya Pradesh Government and Gold Medal from Jadavpur University.

Sanjeev Sharma is doing his Ph.D from ABV- Indian Institute of Information Technology and management Gwalior. He did his $M$. Tech. from ABV- Indian Institute of Information Technology and management Gwalior, India. He received the M. Sc. degree in computer science from jiwaji university Gwalior. His area of research are soft computing, Biometrics, Artificial Intelligence.

Nirupama Tiwari is an Assistant Professor in SRCEM , Banmore. Her field of research includes Data Mining and Warehousing. She has published many research papers in national and international journals/conferences 